\title{
PRODUÇÃO DE MEDICAMENTOS EM FARMÁCIA DE MANIPULAÇÃO: ANÁLISE DA QUALIDADE DOS FÁRMACOS E SUA ESTABILIDADE
}

\author{
Thalita Oliveira ${ }^{1}$ \\ Leonardo Guimarães de Andrade ${ }^{2}$
}

RESUMO: A qualidade dos medicamentos ministrados pelos estabelecimentos apresenta evidencias que geram apreensão para a Agência Nacional de Vigilância Sanitária (ANVISA). De tal modo, as farmácias de manipulados exercem uma enorme função no assunto da Política Nacional de Medicamentos, que visa a aprovação no acesso do uso coerente e a promoção da nação a anódinos eficazes. Tendo em vista a seriedade da abordagem da qualidade dos produtos manipulados, este artigo apresenta relevantes informações sobre o perfil de qualidade do serviço de saúde prestado por esse setor e a estabilidade dos medicamentos. A metodologia realizada foi mediante a revisão da legislação e de trabalhos científicos que englobam o assunto.

Palavras-chave: Produtos farmacêuticos manipulados. Controle de Qualidade de fármacos e medicamentos. Política Nacional de Medicamentos.

ABSTRACT: The quality of the products supplied by the establishments has shown a concern for the National Health Surveillance Agency (ANVISA). Therefore, magistral pharmacies play an important role in the context of the National Medicines Policy, which aims to guarantee the promotion of rational use and the population's access to essential medicines. Given the importance of addressing the quality of products handled, this article presents relevant information about the quality profile of the health service provided by this sector and the stability of medicines. The methodology carried out was through the review of legislation and scientific works that encompass the subject.

Keywords: Manufactured pharmaceuticals. Quality control of drugs and medicines. National Medicines Policy.

\footnotetext{
${ }^{I}$ Discente do curso de farmácia pela UNIG-RJ. E-mail: thalitaisar903@gmail.com.

${ }^{2}$ Professor e orientador no curso de farmácia do UNIG.
} 


\section{INTRODUÇÃO}

Inicialmente existiam estabelecimentos precários que eram realmente focados em homeopatia ou dermatologia, enfoco na individualização da prescrição. Com o início da comercialização dos fármacos genéricos, a fração calhou a manejar diversos fármacos cujo aspectos estão disponíveis através da indústria farmacêutica (VASCONCELOS, 20I6).

A ampliação dos algarismos de fármacos manejados no país procedeu em máxima apreensão com a qualidade destes produtos. A Agência Nacional de Vigilância Sanitária (ANVISA) divulgou, em I9 de abril de 2000, a regulamentação primitiva característica para este âmbito, a RDC $\mathrm{N}^{\circ}$ 33. Esta licença estabeleceu as boas práticas de manipulação em farmácias e propiciou significativas evoluções quanto à qualidade dos produtos magistrais (DE ALMEIDA, 2012)

Em 2005, a ANVISA difundiu o conselho público número 3, na qual constituíram debates de pontos falhos na $\mathrm{RDC} \mathrm{N}^{\circ} 33$, com a finalidade de situar juízo crítico mais ríspidos para a manipulação no Brasil. Então criou-se a RDC $\mathrm{N}^{\circ}$ 214, em 12 de dezembro de 2006, em

seguida revogada pela $\mathrm{RDC} \mathrm{N}^{\circ} 67$, de 8 de outubro de 2007 , a qual se depara em potência. A cobrança cada vez mais rígida nos comandos sanitários comprova a inquietação com a qualidade dos produtos manipulados (DE ALMEIDA, 2012)

A questão que fundamenta tal controvérsia é o caso dos estabelecimentos manipuladores apreendem menos abundância dos recursos financeiros em referência à indústria farmacêutica e isto ergue um formidável ponto: os produtos magistrais conseguem manter o mesmo padrão de qualidade dos produtos industrializados?

Objetivando fornecer subsídios para responder a esta questão e promover maior compreensão sobre o papel das farmácias magistrais, este artigo suscita informações relevantes sobre a importância e a qualidade dos produtos manipulados nas farmácias brasileiras. 


\section{METODOLOGIA}

O presente artigo trata-se de um estudo de revisão bibliográfica, no qual foram utilizadas bibliografias já publicadas em forma de livros e artigos científicos em português e línguas estrangeiras que tratam sobre a qualidade dos produtos de farmácias de manipulação.

Desta forma, foi realizado levantamento bibliográfico de artigos científicos indexados nos bancos de dados Bireme (Centro Latino - Americano e do Caribe de Informação e Ciências da Saúde), nas bases de dados Medline (Base de dados da literatura da área médica e biomédica), Scielo (Scientific Eletronic Library Online) e Lilacs (Índice da literatura científica e técnica da América Latina e Caribe), publicados entre os anos de 2015 a 2021. Estabelecendo um levantamento bibliográfico utilizando-se os seguintes descritores: Produtos farmacêuticos manipulados. Controle de Qualidade de fármacos e medicamentos. Política Nacional de Medicamentos.

Com relação aos critérios de inclusão e exclusão, selecionou-se artigos científicos publicados na língua portuguesa e estrangeiras, artigos disponíveis on-line, artigos prospectivos e de campo publicados no período de 2015 a 2021. Foram excluídos os artigos que não se enquadraram na temática abordada, que fugia do objetivo e critérios da pesquisa, artigos que foram publicados hà mais de ıo anos e artigos incompletos. Os dados encontrados foram analisados, comparados e discutidos, permitindo, desta forma, a formulação de considerações importantes sobre o tema abordado neste trabalho.

\section{REFERENCIAL TEÓRICO}

\section{I FARMÁCIA MAGISTRAL}

Define-se a farmácia como um estabelecimento de manipulação de formulas prescritas e oficinais, de mercado de fármacos, drogas, insumos de socorro e correlatos. Uma distinção excepcional de drogaria para farmácia é o fato de que na drogaria não pode suceder a manipulação de remédios, permitindo somente a venda de drogas, remédios, insumos 
farmacopolas e correspondentes em seus recipientes originais, ou seja, industrializados (CONSELHO REGIONAL DE FARMÁCIA, 2018)

Pode-se considerar fórmulas manipuladas aquelas que são desenvolvidas dentro da farmácia de maneira que siga a prescrição de um profissional da saúde habilitado para tal função, de forma que atenda o paciente individualmente e seguindo a sua composição, posologia, forma farmacêutica e modo de usar. Entretanto, as formas oficiais são as descritas no Formulário Nacional da Anvisa ou em outros formulários oficialmente reconhecidos (MOTA, 2017; FORMULÁRIO NACIONAL DA ANVISA, 2020)

Para diferenciação do exercício da farmácia magistral é conciso situar as contestações entre o procedimento artesanal de manipulação de antálgicos e o método industrial. A manipulação magistral pode ser acentuada como a elaboração, combinação, modificação, recipiente e a rotulação de um fármaco ou produto a partir de uma ordem. Já o processo industrial é qualificado pela fabricação, elaboração, convertimento ou processamento de um remédio ou produto em ampla linha graduada, diferenciando lotes, com forma boticária e dose unificada, definida.

Assim sendo, a diferença principal em meio aos medicamentos produzidos numa farmácia de manipulação em afinidade as drogas industrializadas é a adaptação da formulação (dose, quantidade ou forma farmacêutica) a cada paciente. Também é aceito ao prescritor situar cooptações dentre os fármacos diferentes numa mesma formulação (DIAS, 2020; FARIAS, 2020; DE FIGUEIREDO, 2021).

São estimados possíveis acrescimentos do medicamento manuseado, a facilidade posológica, a probabilidade de opção da forma farmacêutica, consentindo ao prescritor a alternativa da formula que mais se acomode à via de comando selecionada ou às categorias do paciente, a probabilidade de desempenhe de medicamentos por meio da manipulação de medicamentos que foram cessados pelos ambulatórios, à moderação, à personalização da 
clínica e à união multiprofissional, qualificada pela trilogia da saúde, o relacionamento entre farmacêutico, médico e paciente (DIAS, 2020).

\subsection{QUALIDADE DOS MEDICAMENTOS}

Existem diversos fatores que influenciam na qualidade dos medicamentos manipulados, nos qual contribuem para a distribuição, produção, uso dos produtos, transporte e desenvolvimento. Por conseguinte, ao debatermos sobre o controle de qualidade dos remédios de manipulação, precisa-se considerar alguns parâmetros, como: a solução dos princípios ativos dentro do permitido, igualdade nos conteúdos de cada dose manipulada, não permitindo a contaminação seja ela cruzada ou microbiológica, aspectos até o momento do uso, eficácia terapêutica e manutenção da potência (BARATA-SILVA, 2017).

Há muitos anos, o controle de qualidade dos fármacos aferia apenas a qualidade dos produtos das matérias-primas utilizadas no processo de produção e nos produtos finais. Nos dias atuais, diversas funções podem ser conferidas ao controle de qualidade como, por exemplo, preparação, revisões das metodologias pré-estabelecidas aos processos, incubação de

particularizações dos ingredientes, materiais do recipiente e produtos finais, cumprimento de apreciações, sustentação e guarda dos apontamentos analíticos, laboração de planos de amostragem, instruções de estudos de equilíbrio, envio de pareceres e sobretudo, a verificação de todas as etapas durante a produção (BARATA-SILVA, 2017; LOMBARDE, 2017).

Na farmácia de manipulação, a produção acontece diante de uma determinação clínica e para um paciente individualizado, não há concepção de lotes e a ampla disparidade de formulações impede a efetivação dos exames de controle de qualidade no produto acabado. Ademais, como são lançadas poucas quantidades por vez, a fabricação extra para a verificação da qualidade com metodologias mais cuidadosas, como os químicos ou biológicos, ou a manutenção de amostras de contraprova, é considerada financeira e tecnicamente difícil (ARAÚJO, 2017). 
Assim, é essencial que nestes mercados grande parte das etapas aplicadas no método sejam monitoradas e controladas, de maneira que garanta a qualidade do produto final. Idealmente, a fabricação de um remédio seguro solicita a comprobação, mediante a experimentos de controle de qualidade, da harmonia com propriedades de conteúdo do princípio ativo por unidade posológica, igualdade, equilíbrio e predicados apropriados, contendo a rotulação (DIAS, 2020; BISOGNIN, 2013).

Existem outros grandes feitios que regulamentam a qualidade do produto acabado. São eles: volume médio, peso, igualdade das porções, onde são analisadas a disseminação ou transformação dos conteúdos por unidade, a solução, equivalem a configuração de liberação do fármaco para ser meditado pelo organismo. Também são acuradas as propriedades físicas e microbiológicas que precisam acatar alcances verificados pelas farmacopeias e bibliografias auxiliares (BISOGNIN, 2013).

O recipiente empregado para armazenar o produto necessita de passar por uma escalação de maneira a garantir amparo versus os fatores ambientais atribulados (umidade, contaminação microbiana, oxigênio, luz solar e outros), imponderações físicas (choque, 383 impacto, perfuração e vibração) durante o armazenamento e transporte, visando principalmente conservar os modelos de qualidade durante o andamento do uso (SILVA, 2017).

Além disso, falando em proteção a rotulação com articulares adequados consente o usufrutuário a inconfundível identificação, rastreabilidade e mantimento do medicamento incluso aos modelos alcançados, além de elementos de direção como ao emprego de produto. O monitoramento da metodologia produtiva manipularmente poderia ser concretizado mediante o comando da propriedade de ingredientes e recipientes, de modelos de produtos acabados de diferentes configurações farmacêuticas, do controle do ambiente de manipulação, entre outros parâmetros que funcionam como indicadores desse processo. 
Para implantação do controle de qualidade, a farmácia magistral, além de atender as exigências legais, deve seguir as seguintes etapas: verificação das especificações de qualidade, levantamento dos métodos analíticos validados, elaboração de procedimentos operacionais padronizados (POP's), levantamento de equipamentos, utensílios e reagentes necessários e planejamento do espaço físico (BISOGNIN, 2013; SILVA, 2017).

\subsection{PRAZOS DE UTILIZAÇÃO DOS MEDICAMENTOS MANIPULADOS}

O prazo de utilização dos medicamentos magistrais é o período no qual o medicamento mantém as características e os padrões de qualidade pré-definidos e pressupõe a utilização do medicamento. Para a sua atribuição deve-se ter em conta que se trata de preparações extemporâneas, cuja sua aplicação deverá ser efetuada num prazo menor de tempo, assim como considerar o tipo de formula farmacêutica, o fármaco, os recipientes e tipos de embalagens (MARQUES, 2014).

A literatura cientifica não disponibiliza dados relativos à estabilidade de um medicamento manipulado, tornam-se imprescindíveis algumas regras para a atribuição de prazos de utilização dos produtos, que se encontram resumidos na tabela I.

Tabela r: Regras gerais para a atribuição dos prazos de utilização dos medicamentos manipulados (FGP).

\begin{tabular}{|c|c|c|}
\hline \multirow{2}{*}{ Preparaçōes sölidas ou soluçōes nảo aquosas } & $\begin{array}{l}\text { Substância activa é obtida através de um } \\
\text { produto industrializado }\end{array}$ & $\begin{array}{l}25 \% \text { do tempo que resta para expirar o prazo de } \\
\text { validade sem exceder os } 6 \text { meses. }\end{array}$ \\
\hline & $\begin{array}{l}\text { Substância activa é obtida através de matéria- } \\
\text {-prima individualizada }\end{array}$ & 6 meses \\
\hline Preparaçoes liquidas aquosas & Substâncias activas no estado sólido & 14 dias (conservaçāo entre $2-8^{\circ} \mathrm{C}$ ) \\
\hline Restantes preparaçöes & $\cdots$ & $\begin{array}{l}\text { deve corresponder à duração do tratamento sem } \\
\text { exceder os } 30 \text { dias }\end{array}$ \\
\hline
\end{tabular}

\subsection{MECANISMOS DE DEGRADAÇÃO}

Quando ocorre algum tipo de instabilidade nos medicamentos, em certos casos, esta pode ser detectada através de alguma mudança na aparência física, como: cor, sabor, odor ou 
textura da fórmula. Por outro lado, as alterações químicas que possam surgir não são tão evidentes e por muitas vezes só se consegue determinar por meio de análises químicas dos compostos.

Muitos fármacos são suscetíveis a algum tipo de decomposição química, quando formulados no estado liquido ou mesmo em formas farmacêuticas solidas. Tal degradação não só leva a uma perda de potência do fármaco, mas pode, em alguns casos, causar alterações na aparência física das fórmulas farmacêuticas, como por exemplo, a descoloração após a decomposição fotoquímica do medicamento.

\subsection{DEGRADAÇÃO QUÍMICA}

A instabilidade química esta sempre associada a uma perda de potência e de qualidade do medicamento. A identificação, o doseamento dos produtos e degradação permitindo um melhor conhecimento da cinética de degradação, assim como, conhecer as condições para melhorar os medicamentos magistrais que apresentam estruturas químicas através de diversos mecanismos tais como: hidrólise, oxidação, racemização, isomerização, desidratação e fotodegradação (MARQUES, 2014). Na tabela 2 estão presentes os principais mecanismos de degradação química.

Tabela 2: Principais mecanismos de degradação química dos medicamentos (adaptado de Espinosa et al.)

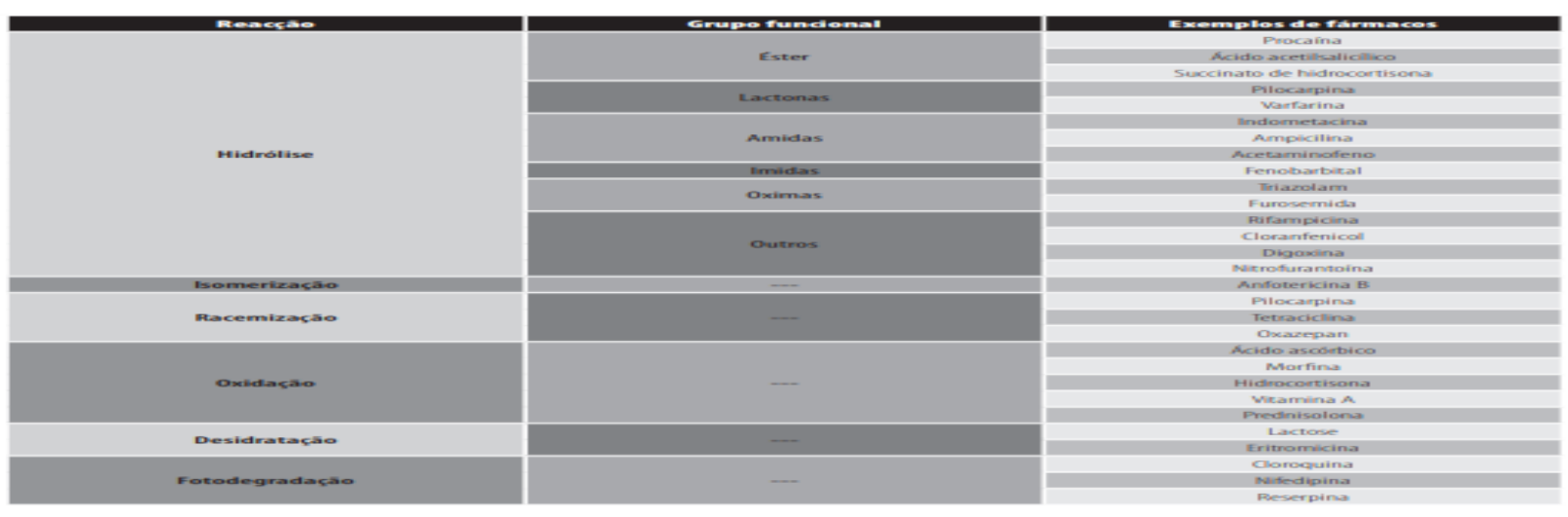




\subsection{A VIGILÂNCIA SANITÁRIA NAS FARMÁCIAS MAGISTRAIS}

A Anvisa é o órgão responsável pelo controle sanitário no Brasil, sendo uma autarquia federal ligada ao Ministério da Saúde com a finalidade de "promover a proteção da saúde da população por intermédio do controle sanitário da produção e da comercialização de produtos e serviços submetidos à vigilância sanitária, inclusive dos ambientes, dos processos, dos insumos e das tecnologias a eles relacionados (DE ALMEIDA, 2012).

O termo controle é usado para os atos sanitários sobre ditos-cujos, celeridades, substâncias, produtos, empregos e assessorias, para que estes não se afastem de leis préestabelecidas. A autoridade inclui alvará, ordem de funcionamento e apontamento, meios usados pela Administração Pública para interferir nas prestezas privadas e as acomodar aos negócios grupais (DE ALMDEIDA, 2012).

Para que a vigilância sanitária consiga desempenhar seus diferentes aprendizados é aproveitado a instrumentação legal, isto é, as normas e cláusulas técnicas constituem os

algoritmos que tem como objetivo garantir os começos do bem-estar evidente, seguindo o desenvolvimento da ciência e da tecnologia.

A Anvisa convidou para um treino, fiscais farmacêuticos de todo o Brasil que agiam nas revistas de drogarias e, sobretudo, para arriscar colocar a unificação de seus atos aduaneiros. Em seguida o andamento do treino a RDC 214 teve algumas atualizações e foi divulgada a RDC 67, que é a lei empregada desde então nas revistas em drogarias magistrais (FORMULÁRIO NACIONAL DA ANVISA, 2020).

Os principais desafios encontrados na fiscalização destes estabelecimentos são a falta de tempo para execução de todas as avaliações e orientações necessárias, os níveis de conhecimento diferenciados entre os inspetores resultando em divergentes entendimentos legais e entraves políticos. 


\subsection{ATUAIS EXIGÊNCIAS DA VIGILÂNCIA SANITÁRIA PARA O CONTROLE DE QUALIDADE NAS FARMÁCIAS MAGISTRAIS}

$\mathrm{Na}$ indústria de fármacos, a aspereza e as cobranças se ativaram com o boletim de legislações inovadoras, diferenciando o comércio industrial de remédios como um dos mais regulamentados. A principal normativa que abona a propriedade dos antálgicos no Brasil através da regulamentação das boas práticas de fabricação é a RDC no 301/2019 (BRASIL. MINISTÉRIO DA SAÚDE, 2019)

Este alvará aboliu a RDC no $17 / 2010$ que foi culpada por amplo impacto para o campo, constituindo as novas diretrizes para a concretização das verificações sanitárias efetivadas pelos órgãos regulatórios, cada vez mais indiscutíveis. Para que as indústrias farmacêuticas competissem tal alvará, foi cogente uma atualização nas alas de produção, sendo o apontamento de medicamentos exclusivamente consagrados posteriormente a aprovação de qualidades aceitáveis durante a revista regulatória.

A RDC/201930I se tornou cada vez mais compreensiva que a velha RDC 17/2010.

Além de conservar os temas de abonação da propriedade, reivindicações, recolhimento de 387 produto, limpeza individual, auto inspeção e auditoria da qualidade, acomodações e aparelhamentos, propriedade da água empregue na indústria farmacêutica, processos de limpeza e diminuição das infecções cruzadas e microbianas durante a produção, junto a ela foram estabelecidas I4 Instruções Normativas (IN) de argumentos característicos, como podemos ressaltar no quadro I.

Se a RDC 17/2010 já apropinquar-se de argumentos mais exclusivos, e proporcionava uma cobrança máxima para a produção de uma droga industrializada, quando conferida à RDC 67/2007 para elaborações manipuladas, a RDC 301/2019 acarreta uma rigidez com muito detalhamento das Fluxogramas Normativos. Para elucidar determinadas dissensões em meio a estas ambas licenças no Quadro 2 estão as modificações constituídas pela nova legislação e o posicionamento do próprio assunto abordado na RDC 67/2007. 
Revista Ibero- Americana de Humanidades, Ciências e Educação- REASE

Quadro I: Composição das Instruções Normativas e seus respectivos assuntos da RDC 301/2019

\begin{tabular}{|c|c|}
\hline Instruçāo normativa (IN) & Assunto \\
\hline IN $n^{0} 35$ & Medicamentos Estéreis \\
\hline IN $n^{\circ} 36$ & Insumos e Medicamentos Biológicos \\
\hline IN $n^{0} 37$ & Medicamentos Radiofármacos \\
\hline IN $n^{0} 38$ & Gases Substâncias Ativas e Gases Medicinais \\
\hline IN $n^{\circ} 39$ & Medicamentos Fitoterápicos \\
\hline IN $n^{0} 40$ & $\begin{array}{l}\text { Atividades de amostragem de matérias-primas e materiais embalagens utilizados na } \\
\text { fabricaçāo de medicamentos }\end{array}$ \\
\hline IN $n^{\circ} 41$ & Medicamentos Líquidos, Cremes ou Pomadas \\
\hline IN $n^{\circ} 42$ & Medicamentos Aerossóis Pressurizados Dosimetrados para Inalaçāo \\
\hline IN $n^{\circ} 43$ & Sistemas computadorizados utilizados na fabricaçāo de Medicamentos \\
\hline IN $n^{0} 44$ & Radiaçāo Ionizante na Fabricaçāo de Medicamentos \\
\hline IN $n^{\circ} 45$ & Medicamentos Experimentais \\
\hline IN $n^{\circ} 46$ & Medicamentos Hemoderivados \\
\hline IN $n^{\circ} 47$ & Atividades de qualificaçāo e validaçāo \\
\hline IN $n^{0} 48$ & Amostras de referência e de retençāo \\
\hline
\end{tabular}

Quadro 2: Comparação das principais divergências normativas da RDC no 301 de 2019 versus a RDC $\mathrm{n}^{\text {o }}$ 67 de 2007

\section{Atualizaçōes da RDC 301/2019 frente a RDC $17 / 2010$}

Inclusāo das BPF para todo ciclo de vida do produto

Conhecimento completo da cadeia de rastreabilidade do insumo ativo

Introduçāo do Gerenciamento de risco para mitigaçāo de riscos e compartilhamento de área

Auditoria in loco obrigatória para fabricante e fornecedores de insumos ativos

Amostras de referência de todos os lotes de material de embalagem primária e impressa

Monitoramento contínuo de partículas em áreas estéreis grau $\mathrm{A}$

\section{Abordagem da RDC 67/2007}

BPMF estabelecem para as farmácias os requisitos mínimos para a aquisiçāo e controle de qualidade da matéria-prima, armazenamento, manipulaçāo, fracionamento, conservação, transporte e dispensaçāo de preparaçōes magistrais e oficinais

Exigem que os rótulos das matérias-primas fracionadas contenham identificaçāo que permita a rastreabilidade desde a sua origem

Nāo abordam tratamento de gerenciamento de risco

Exigem o estabelecimento de critérios, qualificaçāo dos fornecedores e fabricantes e produtos entregues com certificado de análise

Nāo exigem amostras de referência

Monitoramento periódico do processo de sanitização para detectar o surgimento de microrganismos persistentes ou resistentes

BPF: Boas práticas de fabricação; BPMF: Boas práticas de manipulação em farmácias 


\section{CONCLUSÃO}

A preparação de medicamentos magistrais é uma prática ancestral na atividade farmacêutica e constitui uma realidade atual nas atividades terapêuticas. $O$ conhecimento do processo de fabricação e controle de qualidade das formas galênicas é fundamental para assegurar a qualidade do produto.

Faz-se necessário conhecer a estabilidade e condições de armazenamento de modo a estabelecer um prazo de utilização que permita a manutenção das características iniciais do produto. Atualmente estamos numa era de grande ênfase no que diz respeito à qualidade e documentação de medicamentos manipulados.

Portanto, cabe ao farmacêutico que se dedique à preparação magistral, estar familiarizado e respeitar os regulamentos de qualidade sobre medicamentos manipulados.

\section{REFERÊNCIAS BIBLIOGRAFICAS}

I. ARAÚJO, Kaline Vanessa Martins. Acompanhamento da produção de medicamento e demais produtos manipulados em uma farmácia magistral do município de MossoróRN. 2017.

2. BARATA-SILVA, Cristiane et al. Desafios ao controle da qualidade de medicamentos no Brasil. Cadernos Saúde Coletiva, v. 25, p. 362-370, 2017.

3. BISOGNIN, NAGALI SEGATTO et al. CONTROLE DE QUALIDADE APliCAdo AO MEDiCAMENTO MAGistral. Anais do Salão de Ensino e de Extensão, p. I4I, 2013.

4. BRASIL. MINISTÉRIO DA SAÚDE, 20i9. [Disponível em: http://antigo.anvisa.gov.br/documents/I018I/5389382/\%286\%29RDC_301_2019_COMP .pdf/7d99ico4-e7aI-4957-aed5-3689c62913b2] Acesso em: io de Ago. 202I

5. CAMARA DOS DEPUTADOS, Projeto de lei 43385/1994. [Disponível em: https://www.camara.leg.br/propostas-legislativas/20850] Acesso em: I2 de Ago. 2021 
6. CONCEITO DE FARMÁCIA LEI № 13.021/14 [Disponível em: http://crfce.org.br/2018/o8/27/conceito-de-farmacia-lei-no-I3-021-I4/] Acesso em io de Ago. 202I

7. DE AlMEIDA, Maria Letice Couto; DO NASCIMENTO FILHO, Armando Pereira. Análise e discussão de aspectos críticos da resolução 67/2007 da ANVISA para as farmácias com manipulação. Infarma-Ciências Farmacêuticas, v. 22, n. II/I2, p. 13-24, 2012.

8. DE FIGUEIREDO, Jasmine Garcia; ESTRELA, Maria Amélia Albergaria. COMPARAÇÃO DO TEOR DE IVERMECTINA INDUSTRIALIZADOS E MANIPULADOS POR ESPECTROFOTOMETRIA DE ABSORÇÃO. RECIMA2I-Revista Científica Multidisciplinar-ISSN 2675-6218, v. 2, n. 5, p. e25321e25321, 2021.

9. DE LIMA, Letícia Suyane Bento et al. IMPORTÂNCIA DA FARMÁCIA DE MANIPULAÇÃO NA PEDIATRIA: REVISÃO DE LITERATURA. Revista Multidisciplinar em Saúde, v. 2, n. 3, p. or-or, 202I.

Io. DIAS, Kays Lorena Feitosa; FREY, Jaqueline Almeida; DE OLIVEIRA MARQUEZ Carolinne. AS VANTAGENS DOS MEDICAMENTOS MANIPULADOS X MEDICAMENTOS INDUSTRIALIZADOS: doi. org/10.29327/217514.6. 12-29. Revista Ibero-Americana de Humanidades, Ciências e Educação, v. 6, n. I2, p. Io-ıo, 2020.

II. FARIAS, Fernanda Fernandes et al. Divergências nas exigências regulatórias para preparações magistrais e medicamentos industrializados. Infarma-Ciências Farmacêuticas, v. 32, n. 2, p. 128-136, 2020.

12. FORMULÁRIO NACIONAL DA ANVISA [Disponível em: https://www.gov.br/anvisa/pt-br/assuntos/farmacopeia/formulario-nacional] Acesso em o6 de Ago. 202I 


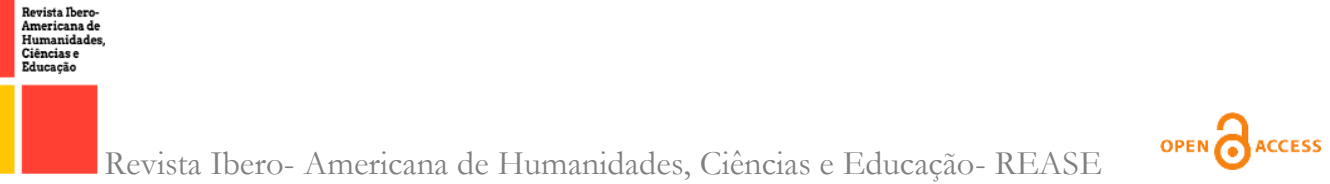

13. LOMBARDO, Márcia; ESERIAN, Jaqueline Kalleian. A análise da qualidade de medicamentos e o papel do laboratório oficial no contexto da saúde pública. Revista de Administração em Saúde, v. 17, n. 67, 2017.

I4. MARQUES, Rodrigo Lopes. Estabilidade dos medicamentos manipulados. 2014.

I5. MOTA, Vivian Aline Mariano; JUNIOR, João Augusto Oshiro; CHIARIANDRÉO, Bruna Galdorfini. O controle da contaminação microbiológica de produtos magistrais. Revista Brasileira Multidisciplinar, v. 20, n. I, p. 33-48, 2017.

16. OLIVEIRA, Luciane Cristina Feltrin de; ASSIS, Marluce Maria Araújo; BARBONI, André René. Assistência farmacêutica no Sistema Único de Saúde: da Política Nacional de Medicamentos à atenção básica à saúde. Ciência \& Saúde Coletiva, v. I5, p. 356I-3567, 2010.

17. SILVA, Ana Sâmula Bezerra da. Estudo do processo produtivo de medicamentos manipulados. 2019.

I8. VASCONCELOS, Thalles Yuri L. et al. A farmácia clínica no âmbito da farmácia magistral. J Appl Pharm Sci [Internet], v. 3, p. 4-6, 2016.

19. VASCONCELOS, Daniela Moulin Maciel de et al. Política Nacional de 391 Medicamentos em retrospectiva: um balanço de (quase) 20 anos de implementação. Ciência \& Saúde Coletiva, v. 22, p. 2609-2614, 2017. 